\author{
Benjamin P. Westley \\ Philip A. Chan
}

\section{Questions remain regarding mandatory use of macrolides in community-acquired pneumonia}

Accepted: 2 May 2010

Published online: 15 July 2010

(C) Copyright jointly held by Springer and ESICM 2010

A reply to this comment is available at: doi:10.1007/s00134-010-1948-8.

Dear Editor,

We read with interest the article by

Martin-Loeches and colleagues [1]

regarding combination antimicrobial therapy for severe community-

acquired pneumonia (CAP). After many years of hotly contested debate, definitive evidence that a specific component of therapy confers survival advantage would be welcome news indeed. However, several findings reported in their study highlight the limitations of observational design and reinforce the need for a randomized trial if this debate is truly to be resolved.

The authors define CAP as an infiltrate on chest radiograph with confirmatory clinical findings that was acquired outside a hospital. However, this does not necessarily exclude healthcare-associated pneumonias (HCAP). According to the 2005 IDSA/ATS guidelines, HCAP and associated multi-drug resistant (MDR) organisms should be considered in the following cases:

antimicrobial therapy in the preceding 90 days; a high frequency of antibiotic resistance in the community; hospitalization for 2 days or more in the preceding 90 days; residence in a nursing home or extended care facility; receipt of home infusion therapy; chronic dialysis; home wound care; or a family member with an MDR pathogen [2]. A significant number of patients in this cohort (10.8\%) were found to have Pseudomonas pneumonia, an atypical cause of CAP. Inclusion of patients with HCAP may have significant ramifications if these patients were more likely to receive a particular treatment regimen. Furthermore, background resistance patterns were not mentioned. High rates of quinolone resistance have been reported in some communities and may have further affected outcomes [3].

The 2005 ATS/IDSA guidelines suggest initial therapy for HCAP include an anti-pseudomonal cephalosporin, carbapenem, or beta-lactam/ beta-lactamase inhibitor, plus either an anti-pseudomonal fluoroquinolone or aminoglycoside, plus either linezolid or vancomycin [2]. Macrolide use is suggested as an alternative to a quinolone if Legionella species are suspected. Compliance with IDSA/ ATS guidelines was required for inclusion in the mortality analysis, and it would be valuable to know if patients with HCAP were over-represented in the quinolone group. A significant number of individuals had co-morbidities (18.3\% with COPD, $15.1 \%$ with diabetes, $24.3 \%$ with cardiomyopathy) that likely placed them at increased risk of HCAP versus CAP.

The data suggest that patients receiving macrolide-containing regimens were quite different from those receiving quinolones. Only five patients receiving macrolides were given an anti-pseudomonal beta-lactam (representing 10.8\% of the group); none received carbapenems. In contrast, in the quinolone group, 32 patients (representing $59.2 \%$ of the group) received either a fourth-generation cephalosporin $(11.1 \%)$, carbapenem $(22.2 \%)$, or co-formulated piperacillin/tazobactam (25.9\%). By direct extrapolation from the 2007 IDSA/ATS CAP guidelines [4], Pseudomonas infection appears to have been suspected in a great deal more patients chosen to receive quinolone therapy. It should not be surprising that mortality would be higher in patients suspected of possible Pseudomonas or MDR infection.

Despite the authors' best attempts to apply adjustments for etiology and severity via Cox regression analysis, we remain unconvinced that these data mandate the use of macrolides in all cases of severe communityacquired pneumonia [5].

\section{References}

1. Martin-Loeches I, Lisboa T, Rodriguez A, Putensen C, Annane D, GarnachoMontero J, Restrepo MI, Rello J (2010)

Combination antibiotic therapy with macrolides improves survival in intubated patients with communityacquired pneumonia. Intensive Care Med 36:612-620. doi:10.1007/s00134009-1730-y

2. American Thoracic Society; Infectious Diseases Society of America (2005) Guidelines for the management of adults with hospital-acquired, ventilatorassociated, and healthcare-associated pneumonia. Am J Respir Crit Care Med 171:388-416

3. Low DE (2004) Quinolone resistance among pneumococci: therapeutic and diagnostic implications. Clin Infect Dis 38(Suppl 4):S357-S362

4. Mandell LA, Wunderink RG, Anzueto A, Bartlett JG, Campbell GD, Dean NC, Dowell SF, File TM, Musher DM, Niederman MS, Torres A, Whitney CG (2007) Infectious Diseases Society of America/American Thoracic Society consensus guidelines on the management of community-acquired pneumonia in adults. Clin Infect Dis 44(Suppl 2):S27-S72

5. Waterer GW (2010) Are macrolides now obligatory in severe community-acquired pneumonia? Intensive Care Med 36:562-564

B. P. Westley $(\bullet)$ P. A. Chan Division of Infectious Disease, Department of Medicine, Alpert Medical School of Brown University, Providence, RI, USA e-mail: bwestley@lifespan.org 